\title{
FAUNA EDÁFICA EM PASTAGEM PERENE SOB DIFERENTES FONTES DE NUTRIENTES ${ }^{(1)}$
}

\author{
Eliana Vera Geremia ${ }^{(2)}$, Julia Corá Segat ${ }^{(3)}$, Ivandro Antonio Fachini ${ }^{(4)}$, Edir Oliveira da \\ Fonseca $^{(5)}$, Dilmar Baretta ${ }^{(5)}$ \\ (1) Trabalho realizado na Escola Básica Demétrio Baldissarelli, Chapecó, SC. \\ (2) Graduanda do Curso de Zootecnia, Centro de Educação Superior do Oeste - CEO/UDESC. Bolsista de Iniciação Científica \\ PIBIC/UDESC. \\ (3) Mestranda da Escola Superior de Agricultura "Luiz de Queiroz". \\ (4) Graduando do Curso de Zootecnia, Centro de Educação Superior do Oeste - CEO/UDESC. \\ (5) Professor do Departamento de Zootecnia, Centro de Educação Superior do Oeste - CEO/UDESC.
}

\begin{abstract}
RESUMO: O presente estudo objetivou avaliar o efeito de fontes de adubação em pastagem de Tifton 85 sobre a fauna edáfica e a relação desta com as variáveis físicoquímicas do solo. $\mathrm{O}$ experimento foi conduzido em um Latossolo testando três fontes de adubação: 1) orgânica com aplicação de adubo orgânico a base de cama de aves $(\mathrm{O}) ; 2$ ) orgânica + mineral $\mathrm{O}+$ fertilizante mineral $(\mathrm{OM})$ e 3 ) adubação mineral $(\mathrm{M})$. $\mathrm{O}$ delineamento experimental foi constituído de blocos ao acaso com três repetições. A coleta para avaliação da fauna edáfica foi realizada por armadilhas do tipo "Trampas de Tretzel" e, nos mesmos pontos, foram coletadas amostras de solo para análise dos atributos químicos, mensurada a resistência à penetração e umidade do solo. Houve relação entre a abundância de grupos da fauna edáfica com algumas variáveis ambientais e físico-químicas do solo, especialmente a umidade, matéria orgânica, potássio e fósforo. $\mathrm{O}$ tratamento $\mathrm{O}$ proporcionou maiores valores de abundância e diversidade da fauna edáfica, não sendo a abundância diferente do tratamento OM.
\end{abstract}

Palavras-chave: Bioindicadores, fauna do solo, sustentabilidade, análise multivariada.

\section{SUMMARY: EDAPHIC FAUNA IN PASTURES UNDER DIFFERENT PERENNIAL NUTRIENT SOURCES}

\begin{abstract}
This study aimed to evaluate the effect of fertilizer sources grazing Tifton 85 on soil fauna and its relation to the physical-chemical parameters of the soil. The experiment was conducted in a Oxisol testing three fertilization sources: 1) organic with application of organic manure the birds bed base $(\mathrm{O}) ; 2)$ organic + mineral $\mathrm{O}+$ mineral fertilizer $(\mathrm{OM})$ and 3) mineral fertilizer (M). The experimental design consisted of randomized blocks with three
\end{abstract}


replications. The collection for evaluation of soil fauna was held by traps such as "Tretzel tramps" and, at the same points, soil samples were collected for analysis of chemical attributes, measured penetration resistance and soil moisture. There was a relationship between the abundance of soil fauna groups with some environmental variables and physicochemical soil, especially moisture, organic matter, potassium and phosphorus. The treatment gave the highest values of abundance and diversity of soil fauna, not being different plenty of OM treatment.

Key-words: Bioindicators, soil fauna, sustainability, multivariate analysis.

\section{INTRODUÇÃO}

As pastagens constituem a principal fonte de alimento para o rebanho brasileiro de ruminantes domésticos, o que tem propiciado elevada competitividade no mercado internacional de carne e leite, em razão do baixo custo de produção, especialmente com alimentação (SILVA et al., 2008).

Em áreas exploradas com pastagens perenes deve-se buscar a melhor combinação de manejo, adubação e gerenciamento das atividades, tanto no verão como no inverno, de forma que a produção animal não prejudique a produção vegetal. Entretanto, quando não manejadas adequadamente pode resultar em aumento da perda de água por evaporação, perdas de nutrientes por erosão e aumento da compactação do solo e especialmente diminuição da diversidade da fauna edáfica (AMORIM et al., 2013; ROSA et al., 2015).

Pode-se destacar como fontes de adubação da pastagem de Tifton 85 (Cynodon spp), a aplicação de adubos orgânicos. De maneira geral, essa fonte favorece a manutenção da matéria orgânica do solo, melhorando suas propriedades físicas, químicas e biológicas. A matéria orgânica auxilia a atividade dos organismos do solo, que por sua vez resulta em impactos positivos sobre a ciclagem de nutrientes (ALVES et al., 2008; BRONW; SAUTTER, 2009; MURCHIE et al., 2015). Do ponto de vista físico, o uso de adubos orgânicos promove o aumento da estabilidade de agregados, associado à redução da densidade do solo (ANDREOLA et al., 2000). A macrofauna do solo tem importante papel nos processos do ecossistema no que concerne à ciclagem de nutrientes e estrutura do solo, pois é responsável pela fragmentação dos resíduos orgânicos, mistura das partículas minerais e 
orgânicas, redistribuição da matéria orgânica, além de produzir "pellets fecais" (BARETTA et al., 2011; PAULA; LOPES, 2013; SHUKLA et al., 2013).

Dentre as diferentes formas de monitorar a qualidade do solo em uma pastagem de Cynodon spp. cv. Tifton 85 destaca-se o uso de indicadores biológicos, especialmente a avaliação da fauna edáfica (BARETTA et al., 2003, PEREIRA et al., 2013).

Os organismos edáficos possuem a capacidade de dar respostas rápidas a mudanças na qualidade do solo, característica que não é observada nos indicadores físico-químicos. Em alguns casos, alterações na abundância, diversidade e na atividade de organismos edáficos, incluindo microrganismos, podem indicar mudanças nas propriedades físicas e químicas, refletindo um claro sinal na melhoria ou degradação da qualidade do solo (VASCONCELLOS et al., 2013).

Neste contexto, a adição de resíduos orgânicos em uma pastagem de Tifton 85 é um fator que pode influenciar a biota do solo, principalmente pelo fornecimento de alimento para os organismos edáficos. O presente trabalho objetivou avaliar o efeito das diferentes formas de adubação sobre a composição da comunidade edáfica do Tifton 85 .

\section{MATERIAL E MÉTODOS}

O experimento foi conduzido na região Oeste de Santa Catarina, na Escola Básica Municipal Agropecuária Demétrio Baldissareli, localizada no distrito de Marechal Bormann, município de Chapecó, SC. O solo da área experimental é classificado como Latossolo Vermelho distroférrico (EMBRAPA, 2006).

O clima da região é caracterizado como mesotérmico úmido com verão quente ( $\mathrm{Cfa}$ ), segundo a classificação de Köppen, com precipitação média anual de $2.039 \mathrm{~mm}$, bem distribuídos ao longo do ano e temperatura média anual em torno de $18^{\circ} \mathrm{C}$, variando mensalmente de 14,1 a $23{ }^{\circ} \mathrm{C}$ (PANDOLFO et al., 2002). A área onde foram alocadas as parcelas do estudo possui 9.846,232 $\mathrm{m}^{2}$ e vem sendo explorada com pastagens perenes e pastejo contínuo desde 2007, com predominância de Cynodon spp. cv. Tifton 85. Entretanto, 
na área experimental é comum a presença, especialmente no inverno, de azevém (Lolium multiflorum L.).

O delineamento experimental foi constituído de blocos, com três repetições. Cada parcela apresenta dimensões de 0,109 ha, totalizando 9 parcelas [3 tratamentos x 3 blocos (3 repetições verdadeiras) $=9]$.

Os tratamentos testados consistiram de três fontes de adubação: 1) "Orgânico" (O): adubo orgânico a base de cama de aves (valores médios de referência da cama de aves 02-0302); 2) orgânica + mineral (Organomineral) com aplicação de $606 \mathrm{~kg} \mathrm{ha}^{-1}$ de adubo $\mathrm{O}$ + fertilizante mineral da fórmula 04-10-10 (OM) visando ajustar as mesmas quantidades de NPK fornecidas pela adubação mineral e 3) Mineral (M), conforme a recomendação da CQFS RS/SC (2004) para Tifton 85. O fertilizante mineral foi aplicado na quantidade de $150 \mathrm{~kg} \mathrm{ha}^{-1}$ de nitrogênio (N) (aplicado na forma de uréia com 44\% de $\mathrm{N}$ ), $60 \mathrm{~kg} \mathrm{ha}^{-1}$ de fósforo (P) (aplicado na forma de superfosfato triplo com $41 \%$ de $\mathrm{P}_{2} \mathrm{O}_{5}$ ) e $60 \mathrm{~kg} \mathrm{ha}^{-1}$ de potássio (K) (aplicado na forma de cloreto de potássio com $58 \%$ de $\mathrm{K}_{2} \mathrm{OKCl}$ ).

As fontes de adubação foram aplicadas a lanço na superfície do solo. Procurou-se ajustar as três fontes de adubação buscando fornecer as mesmas quantidades de N, P e K para os tratamentos O, OM e M. Após a aplicação das fontes de adubação foi realizada escarificação em cada parcela experimental.

A avaliação da fauna edáfica foi realizada dois meses após a aplicação das fontes de adubação, por meio da instalação de três armadilhas do tipo "Trampas de Tretzel" distribuídas, ao acaso, em cada bloco $[n=3$ blocos x 3 armadilhas por bloco =9]. As armadilhas foram constituídas por frascos de vidro com $6 \mathrm{~cm}$ de diâmetro, enterrados no solo com a extremidade vazada nivelada com a superfície do solo, permanecendo no local por 3 dias em cada tratamento. Cada armadilha foi preenchida com $200 \mathrm{~mL}$ de solução detergente, na concentração de 1,5 \% (BARETTA et al., 2003).

Após a retirada das armadilhas, foi realizado o preparo, a limpeza e a classificação dos organismos edáficos. Inicialmente, as amostras foram passadas em peneiras de $2 \mathrm{~mm}$ e 0,125 $\mathrm{mm}$, separando-se o solo e os fragmentos vegetais dos organismos. Após esta separação os 
organismos foram armazenados em recipientes com álcool etílico a $80 \%$. Posteriormente, foram separados e identificados até p maior nível taxonômico possível, com auxílio de microscópio estereoscópico com aumento de 100x.

Nos mesmos pontos e época de amostragem da fauna do solo, também foram retiradas amostras para análise dos atributos químicos do solo, conforme metodologia descrita em Tedesco et al. (1995) e a resistência do solo à penetração foi avaliada por meio de equipamento de medição automatizada "Solo Track" modelo PLG 5200, e o teor de umidade do solo através do medidor eletrônico de umidade do solo "HidroFarm" modelo HFM 2010.

A partir do resultado do número total de organismos de solo capturados por armadilhas (abundância), foi calculado o índice de diversidade de Shannon $(H)$ através da relação $(H=-$ $\sum$ pi. $\log$ pi), onde: $\mathrm{pi}=\mathrm{ni} / \mathrm{N} ; \mathrm{ni}=$ densidade de cada grupo, $\mathrm{N}=$ número total de grupos) e uniformidade de Pielou (e) (Odum, 1983). Os atributos ecológicos fauna do solo (abundância, diversidade de Shannon $\mathrm{H}$ e uniformidade de Pielou) foram submetidos à análise de variância (ANOVA) e as médias comparadas por meio do teste de LSD $(P<0,05)$, utilizando-se o programa estatístico SAS versão 8.2 (SAS, 2002).

A abundância (número total de organismos capturados $\operatorname{armadilha}^{-1}$ ) de cada grupo taxonômico da fauna do solo nos diferentes tratamentos foi utilizada para a obtenção do comprimento do gradiente. Como este comprimento foi maior que quatro desvios padrão (>4SD = resposta unimodal), optou-se pela Análise de Correlação Canônica (ACC), usando o programa CANOCO versão 4.0 (TER BRAAK; SMILAUER, 1998). Os atributos químicos (pH água, pH SMP, MO, P, K, Ca e Mg), resistência a penetração e a umidade do solo foram utilizados como variáveis ambientais explicativas das modificações dos grupos da fauna do solo (variáveis resposta). Adicionalmente, também se realizou a análise de agrupamento (Cluster Analysis) pelo método de ligação completa (vizinho mais distante) (EVERITT, 1993). Neste caso, foi tomada a "Distância Euclidiana" (EVERITT, 1993; BARETTA et al., 2003), entre o número de grupos capturados (abundância) de cada grupo taxonômico como medida de similaridade, por meio do programa STATISTICA 6.0 (STATSOFT, 2004). A frequência relativa dos grupos também foi calculada para cada tratamento. 


\section{RESULTADOS E DISCUSSÃO}

\subsection{ABUNDÂNCIA DA FAUNA EDÁFICA}

Analisando a abundância (número total de organismos $\operatorname{armadilha}^{-1}$ ) dos grupos da fauna do solo em cada tratamento, nota-se que os tratamentos Orgânico (O) e Organomineral $(\mathrm{OM})$ diferiram do tratamento Mineral $(\mathrm{M})$, apresentando maiores valores de abundância (Figura 1A). Esta diferença se deve em parte pela maior disponibilidade de material orgânico fornecida pelos tratamentos O e OM (Figura 4), em comparação com o tratamento M.

A diversidade da fauna do solo representada pelo índice de diversidade de Shannon (H) também foi maior no tratamento $\mathrm{O}$ em comparação com os tratamentos $\mathrm{OM}$ e $\mathrm{M}$, não sendo estes dois últimos diferentes entre si (Figura 1B). Para o índice de Uniformidade de Pielou (e) observou-se que houve o mesmo comportamento do índice H (Figura 1C).

O comportamento, observado nos índices ecológicos de qualidade do solo, evidencia que a adubação orgânica favorece o desenvolvimento dos organismos do solo, devido ao maior aporte de material orgânico no sistema (SCHIEDECK et al., 2010; BARETTA et al., 2011), promovendo um efeito benéfico sobre os grupos mais frequentes da fauna de solo, corroborando com os dados obtidos por outros autores em estudos Catarinenses pois, além do maior aporte de nutrientes ao solo, representa fonte alimentar adicional para os organismos edáficos (BARETTA et al., 2003; ALVES et al., 2008). 

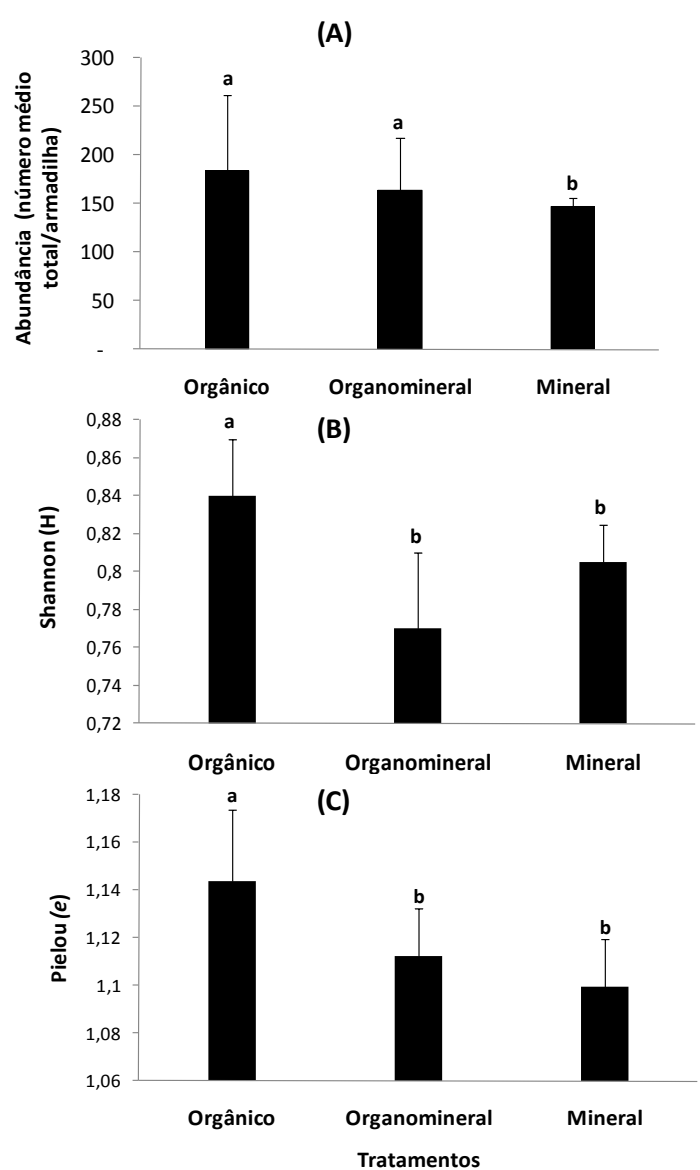

Figura 1. Valores de abundância (número total de organismos armadilha ${ }^{-1}$ ) (A), índices de diversidade de Shannon (H) (B) e uniformidade de Pielou (e) (C), nos tratamentos estudados. $(\mathrm{n}=3$ blocos x 3 armadilhas em cada bloco $=9)$. $(\top)$ Desvios padrão. Médias com a mesma letra na barra não diferem estatisticamente $(P \geq 0,05)$ pelo teste LSD.

Alves et al. (2008) avaliando tratamentos similares aos do presente estudo, os autores constataram efeito negativo sobre a abundância da macrofauna edáfica na presença de adubação mineral e, um efeito benéfico nos tratamentos que receberam fertilizante mineral e orgânico. Esses mesmos autores encontraram maiores valores de índice H no tratamento com adubação orgânica em comparação com os tratamentos organomineral e mineral. Melhorias na qualidade da fauna também foram relatadas por Segat et al. (2015) e Maccari et al. (2016) utilizando dejeto líquido de suínos. 


\subsection{FREQUÊNCIA RELATIVA DA FAUNA EDÁFICA}

A frequência relativa dos grupos Coleoptera, Araneae, Collembola e Isopoda foi semelhante entre os três tratamentos estudados (Figura 2). As formigas (Hymenoptera) foram mais frequentes no tratamento orgânico $(\mathrm{O})$, seguido pelo tratamento mineral (M) representando mais de $15 \%$ da ocorrência total, enquanto no tratamento organomineral (OM) as formigas representaram menos de $15 \%$ da frequência relativa. De acordo com McGlynn; Poirson (2012) esse grupo tem forte relação com atributos químicos e para Dunxião et al. (1999) a presença deste grupo de indivíduos está relacionada a elevada disponibilidade de fósforo, potássio e presença de material orgânico.

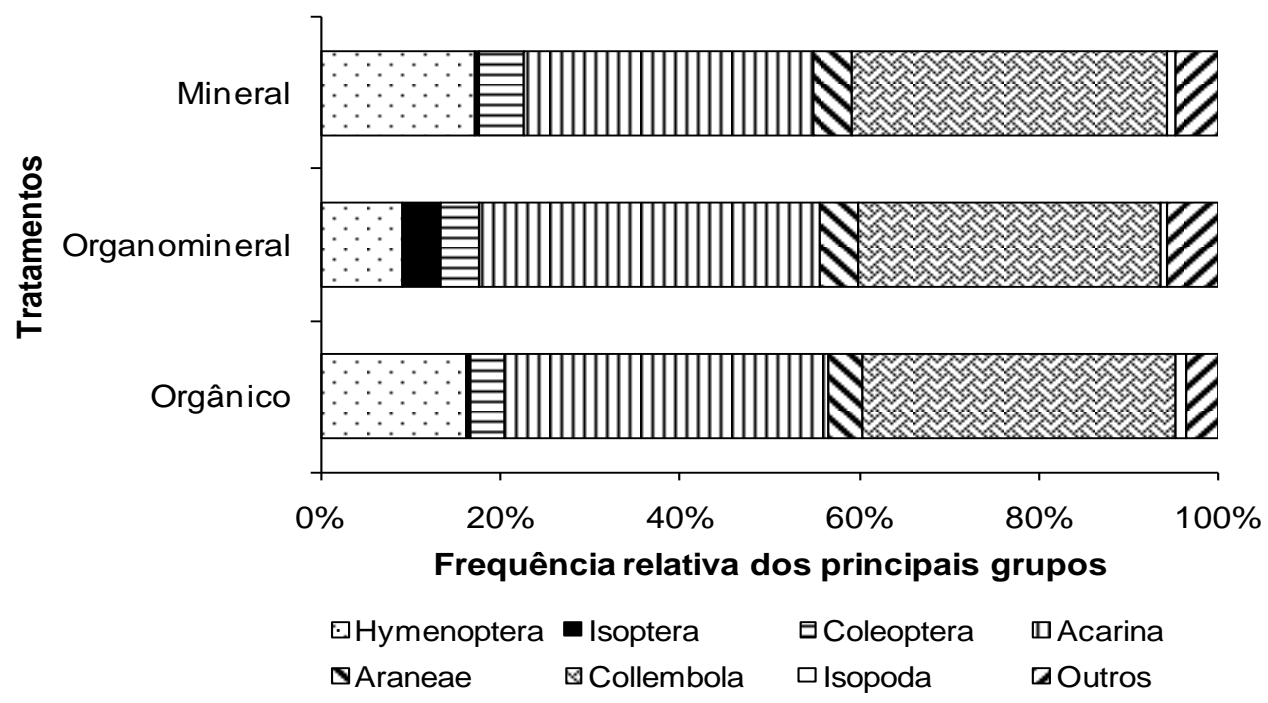

Figura 2. Frequência relativa dos principais grupos da fauna edáfica capturados em cada um dos três tratamentos estudados.

O tratamento OM favoreceu a população de cupins (Isoptera), em comparação aos demais tratamentos. A presença deste grupo de organismos edáficos em pastagens pode estar associada à capacidade destes em obter eficientemente nutrientes da serapilheira de gramíneas (AQUINO et al., 2008; BARETTA et al., 2011).

A ocorrência de ácaros (Acarina) foi maior nos tratamentos $\mathrm{O}$ e $\mathrm{OM}$ com aproximadamente $20 \%$ da ocorrência total. Esse mesmo comportamento foi observado para o grupo Collembola, que foi mais frequente no tratamento $\mathrm{O}$, seguido do tratamento $\mathrm{OM}$. $\mathrm{O}$ 
contrário foi verificado para a ordem Coleoptera, em que a maior frequência foi encontrada no tratamento M. A população de aranhas (Araneae), não apresentou diferença entre os três tratamentos. $\mathrm{O}$ grupo Isopoda teve maior frequência no tratamento $\mathrm{O}$ em relação aos demais tratamentos. A ocorrência de Outros (Thysanoptera; Lepidoptera; Larvas; Diplopoda; Griloblattodea; Hemiptera; Homoptera; Orthoptera; Diplura; Dermaptera; Protura) foi maior no tratamento $\mathrm{OM}$, não diferindo entre os tratamentos $\mathrm{M}$ e $\mathrm{O}$.

\subsection{ANÁLISE DE AGRUPAMENTO DA FAUNA EDÁFICA}

Nas Figuras 3A e 3B são apresentados os dendrogramas obtidos pela análise de agrupamento, onde os números no eixo vertical representam a "Distância Euclidiana" entre grupos taxonômicos mais importantes em termos de abundância que geraram os agrupamentos (Figura 3A) e os tratamentos estudados (Figura 3B). Nesta análise, evidenciouse similaridade entre os grupos taxonômicos Griloblattodea, Isopoda, Orthoptera, Lepidoptera, Diplura, Dermaptera, Hemiptera, Outros (soma de organismos menos frequentes), Protura, Larvas (soma de outras larvas não identificadas), Homoptera, Diplopoda, Chilopoda e Thysanoptera (Figura 3A). Esses grupos se isolaram de Collembola e Acarina que formaram um agrupamento separado com maiores valores de Distância Euclidiana, seguidos por Hymenoptera, Araneae e Coleoptera respectivamente.

Em trabalho realizado no Oeste de Santa Catarina, resultado semelhante foi encontrado em relação ao mesmo comportamento na ordem Collembola que também se isolou das demais ordens (BARETTA et al., 2003). Buscando verificar quais são os tratamentos mais similares entre si em termos de abundância da comunidade da fauna edáfica, evidenciou-se que o tratamento $\mathrm{O}$ apresentou maior Distância Euclidiana e ficou isolado dos tratamentos OM e M, sendo que estes dois últimos formaram agrupamentos com valores muito semelhantes em termos de abundância e composição de grupos taxonômicos. 

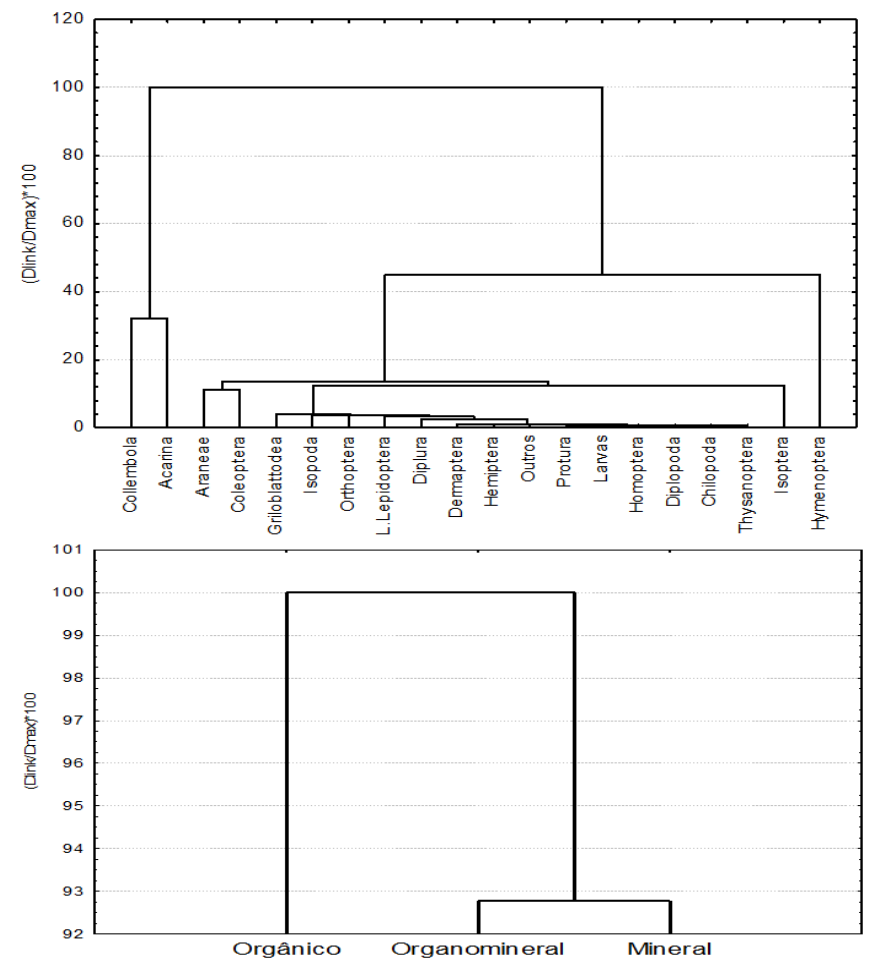

Figura 3. Dendrogramas obtidos pela análise de agrupamento, discriminando os grupos da fauna edáfica (A) e os tratamentos estudados (B), por meio da "Distância Euclidiana" da abundância de grupos capturados da fauna do solo como medida da similaridade. $(n=3$ blocos $x 3$ armadilhas em cada bloco $=9)$.

\subsection{ANÁLISE DE CORRELAÇÃo CANÔNICA (ACC) DA FAUNA EDÁFICA}

A análise de correlação canônica (CCA) para a fauna edáfica demonstrou que houve separação entre as três fontes de adubação e uma relação significativa $(P<0,05)$ entre a abundância da fauna edáfica e os atributos físico-químicos do solo analisados. Os grupos Hymenoptera, Araneae, Homoptera e Outros ficaram fortemente associados ao tratamento O, enquanto os grupos Isoptera, Larvas, Thysanoptera, Dermaptera e Lepidoptera com o tratamento OM. Já os grupos Isopoda, Orthoptera e Coleoptera ficaram mais associados com o tratamento $\mathrm{M}$, contribuindo de forma mais expressiva que os grupos para separar o tratamento $\mathrm{M}$ dos demais tratamentos $(\mathrm{O}$ e $\mathrm{OM})$.

Quando os atributos químicos do solo foram utilizados como variáveis ambientais explicativas, evidenciou-se que eles auxiliaram na interpretação e na discussão dos resultados (Figura 4). Nesse sentido, os grupos Hymenoptera, Araneae e Homoptera e Outros no tratamento $\mathrm{O}$ obtém sua máxima abundância quanto maiores forem os valores de matéria 
orgânica (MO) e umidade do solo. Da mesma forma, quando os tratamentos estudados se apresentam em quadrantes separados, indica que a fauna e as variáveis ambientais ocorrem de forma diferenciada nestes tratamentos.

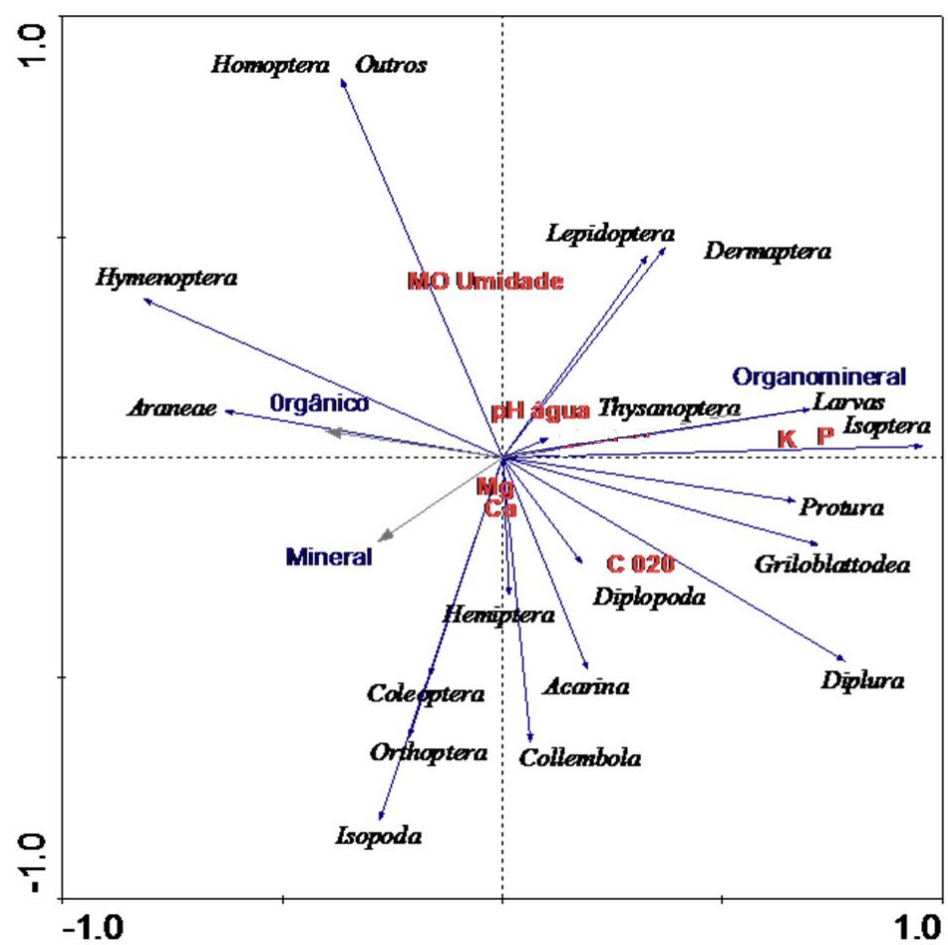

Figura 4. Resultado da relação entre as dimensões 1 e 2 da análise canônica de correlação canônica (ACC) para a abundância da fauna edáfica e atributos físico-químicos do solo nos tratamentos estudados. pH: Potencial hidrogeniônico; MO: Matéria Orgânica; P: Fósforo; K: Potássio; Ca: Cálcio; Mg: Magnésio e C_0-20: resistência a compactação na profundidade $0-20 \mathrm{~cm}$.

Pode-se observar também, que os atributos químicos Fósforo (P) e Potássio (K) apresentaram-se fortemente associados ao tratamento OM (Figura 4). Os demais atributos fisico-químicos do solo ( $\mathrm{pH}$ água, $\mathrm{pH} \mathrm{SMP}, \mathrm{Mg}$ e $\mathrm{Ca}$ e a resistência a penetração na profundidade de 0-20 cm) não contribuíram substancialmente para explicar as modificações da comunidade da fauna edáfica e não se associaram especificamente a nenhum dos tratamentos, uma vez que estão dispostos na porção mediana (valores menores e/ou próximos a zero) ou em quadrantes diferentes de onde os tratamentos se encontram (Figura 4). 


\section{CONCLUSÕES}

A abundância e a diversidade da fauna edáfica foram sensíveis indicadores da qualidade do solo em pastagem de Tifton 85 .

A Adubação orgânica e organomineral proporciona abundância e diversidade da fauna edáfica, similar entre os tratamentos.

Houve maior sustentabilidade da pastagem de Tifton 85, especialmente em termos de manutenção de indicadores biológicos de qualidade do solo quando a pastagem recebeu adubação orgânica, em comparação com as adubações organomineral e mineral.

\section{AGRADECIMENTOS}

Os autores agradecem à bolsa PROBIC/UDESC; a Prefeitura Municipal de Chapecó e aos técnicos da Escola Básica Municipal Agropecuária Demétrio Baldissareli pela disponibilidade da área e ajuda; aos graduandos Ederson A. Andrade, Felipe V. Zandavalli e Tádia E. Stivanin pela ajuda; ao Departamento de Zootecnia e ao funcionário Gilberto François do CEO/UDESC; a empresa FERTICEL e Agrisus (Bolsista I. A. Fachinni) pelo apoio. D. Baretta agradece ao CNPq (Conselho Nacional de Desenvolvimento Científico e Tecnológico) pela Bolsa de Produtividade Científica do CNPq (Processo no $307162 / 2015-0$ ).

\section{Referências}

ALVES, M. V.; SANTOS, J. C.; GOIS, D. T.; ALBERTON, J. V.; BARETTA, D. Macrofauna do solo influenciada pelo uso de fertilizantes químicos e dejetos de suínos no Oeste do Estado de Santa Catarina. Revista Brasileira de Ciência do Solo, Viçosa, v. 32, p. 589-598, 2008.

AMORIM, I. A.; AQUINO, A. L.; SILVA, E. M. J.; MATOS, T. E. S.; SILVA, T. P.; RODRIGUES, D. M. Levantamento de artrópodes da superfície do solo em área de pastagem no assentamento Alegria, Marabá-PA. Agroecossistemas, sl. v, 5, p. 62-67, 2013.

ANDREOLA, F.; COSTA, L. M.; OLSZEVISKI, N. Influência da cobertura vegetal de inverno e da adubação orgânica e ou mineral sobre as propriedades físicas de uma terra roxa estruturada. Revista Brasileira de Ciência do Solo, Viçosa, v. 24, p. 857-865, 2000. 
AQUINO, A. M.; CORREIA, M. E. F.; ALVES, M. V. Diversidade da Macrofauna Edáfica no Brasil. In: MOREIRA, F. M. S; SIQUEIRA, J. O.; BRUSSAARD, L. Biodiversidade do Solo em Ecossistemas Brasileiros. Lavras - MG, Ed. UFLA. p.142-170, 2008.

BARETTA, D.; SANTOS, J. C. P.; MAFRA, Á.L.; WILNDER, L. P.; MIQUELLUTI, D. J. Fauna edáfica avaliada por armadilhas de catação manual afetada pelo manejo do solo na região oeste catarinense. Revista de Ciências Agroveterinárias, Lages, v. 2, p. 97-106, 2003.

BARETTA, D., SANTOS, J. C. P., SEGAT, J. C., GEREMIA, E. V., DE OLIVEIRA FILHO, L. C. I., ALVES, M. V. Fauna edáfica e qualidade do solo, in: KLAUBERG FILHO, O., MAFRA, A. L., GATIBONI, L. C. (Eds), Tópicos especiais em ciência do solo, Sociedade Brasileira de Ciência do Solo, Viçosa, 2011, pp. 141-192.

BROWN, G.G.; SAUTTER, K. D. Biodiversity, conservation and sustainable management of soil animals: the XV International Colloquium on Soil Zoology and XII International Colloquium on Apterygota. Pesquisa Agropecuária Brasileira, Brasília, v. 44, p. 1-9, 2009.

COMISSÃO DE QUÍMICA E FERTILIDADE DO SOLO - CQFSRS/SC. Manual de adubação e de calagem para os estados do Rio Grande do Sul e Santa Catarina. 10. ed. Porto Alegre, SBCS/NRS, 2004. 400p.

DUNXIÃO, H.; CHUNRU, H.; YALING, X.; BANWANG, H.; LIYUAN, H.; PAOLETTI, M.G. Relationship between Soil Arthropods and Soil Properties in a Suburb of Qianjiang City, Hubei, China. Critical Reviews in Plant Science, sl., v.18, p. 467-473, 1999.

EMBRAPA - CNPS. Sistema Brasileiro de Classificação de Solos. Brasília: EMBRAPA. 2 Ed. Rio de Janeiro, 2006. 412p.

EVERITT, B.S. 1993. Cluster analysis. New York: John Wiley, 1993. 170p.

MACCARI, A. P.; BARETTA, D.; PAIANO, D.; LESTON, S.; FREITAS, A.; RAMOS, F.; SOUSA, J. P.; KLAUBERG-FILHO, O. Ecotoxicological effects of pig manure on Folsomia candida in subtropical Brazilian soils. Journal of Hazardous Materials, sl, v. 314, p. 113-120, 2016.

MCGLYN, T. P.; POIRSON, E. Ants accelerate litter decomposition in a Costa Rican lowland tropical rain forest. Journal of Tropical Ecology, sl, v. 28, p. 437-443, 2012.

MURCHIE, A. K.; BLACKSHAW, R. P.; GORDON, A. W.; CHRISTIE, P. Responses of earthworm species to long-term applications of slurry. Applied Soil Ecology, Amsterdam, v. 96, p. 60-67, 2015.

ODUM, E.P. 1983. Ecologia. Rio de Janeiro: Guanabara, 1983. 434p.

PANDOLFO, C.; BRAGA, H. J.; SILVA JR, V. P. da; MASSIGNAM, A. M., PEREIRA, E. S.; THOMÉ, V. M. R.; VALCI, F.V. Atlas climatológico do Estado de Santa Catarina. Florianópolis: Epagri, 2002. CD-Rom.

PAULA, J. D.; LOPES, A. Jardins de formigas na Amazônia Central: um experimento de campo utilizando cupins vivos como iscas. Acta Amazonica, sl, v. 43, p. 447-454, 2013.

PEREIRA, J. DE M.; BARETTA, D.; BINI, D.; VASCONCELLOS, R. L.; CARDOSO, E. J. B. N. Relationships between microbial activity and soil physical and chemical properties in native and reforested Auraucaria angustifolia forests in the state of São Paulo. Revista Brasileira de Ciência do Solo, Viçosa, v. 37, p. 572-586, 2013.

ROSA, M. G.; KLAUBERG FILHO, O.; BARTZ, M. L. C.; MAFRA, Á. L.; SOUSA, J. P. F. A.; BARETTA, D. Macrofauna edáfica e atributos físicos e químicos em sistemas de uso do solo no planalto catarinense. Revista Brasileira de Ciência do Solo, Viçosa, v. 39, p. 1544-1553, 2015.

SAS INSTITUTE. SAS: User's guide: statistics. 6th ed. Cary: Institute Inc.,2002. 
SCHIEDECK, G.; STRASSBURGER, K. F. S.; SILVEIRA, E. F.; HOLZ, F. P. Alimentação de minhocas: teste de aceitação do alimento. Pelotas: Embrapa Clima Temperado, 2010. p. $1-8$

SEGAT, J. C.; ALVES, P. R. L.; BARETTA, D.; CARDOSO, E, J. B. N. Ecotoxicological evaluation of swine manure disposal on tropical soils in Brazil. Ecotoxicology and Environmental Safety, Paris, v. 122, p. 91-97, 2015.

SHUKLA, R. K.; SINGH, H.; RASTOGI, N.; AGARWAL, V. M. Impact of abundant Pheidole ant species on soil nutrients in relation to the food biology of the species. Applied Soil Ecology, Amsterdam, v. 71, p. 15-23. 2013.

SILVA, H. A.; KOEHLER, H. S.; MORAES, A.; GUIMARÃES, V. D.; HACK, E.; CARVALHO, P. C. F. Análise da viabilidade econômica da produção de leite a pasto e com suplementos na região dos Campos Gerais - Paraná. Ciência Rural, Santa Maria, v. 38, p. 445-450, 2008.

STATSOFT. STATISTICA 7.0 (data analysis software system). [s.1]. 2004. Disponível em: $<$ http//:www.statsoft.com> Acesso em: 15 fev. 2016.

TEDESCO, M. J.; GIANELlO, C.; BISSANI, C. A.; BOHNEN, H.; VOLKWEISS, S. J. Análises de solo, planta e outros materiais. 2. ed. Porto Alegre: Departamento de Solos da UFRGS, 1995. 174p. (Boletim Técnico, 5).

TER BRAAK, C. J. F.; SMILAUER, P. CANOCO reference manual and user's guide to Canoco for Windows: software for canonical community ordination (version 4). New York: Microcomputer Power, 1998.

VASCONCELLOS, R. L. F.; BONFIM, J. A.; ANDREOTE, F. D.; MENDES, L. W.; BARETTA, D.; CARDOSO, E. J. B. N. Microbiological indicators of soil quality in a riparian forest recovery gradient. Ecological Engineering, sl, v. 53, p. 313-320, 2013. 